\title{
On the World Co2 Emission Equitable Transition Design and Its Implementation: Theory and Empirical Data
}

\author{
By Demetrio Miloslavo Bova ${ }^{1}$
}

\begin{abstract}
This paper designs the transition trajectory for $\mathrm{Co} 2$ emissions that considers the impact on inequality both across countries and generations and introduces elements for its implementation. The intercountry and intergenerational equities are discussed and found, for the first, in terms of equal cumulated per capita emissions, for the second, both in terms of temperature growth control and capacity to ensure an equal future yearly amount of emission per generation. These definitions allow enquiring which country is behaving fairly and, consequently, which system may be introduced to compensate. Once the emission trajectory is defined, its implementation and the deviances management can be structured through a quasi-decentralised dynamic cap and trade system. Theory and empirical data for the design and its management are provided.
\end{abstract}

Keywords: Equitable transition, Inequality of transition, Co2 emissions, Climate justice, Quasi-decentralised dynamic cap and trade.

\section{Introduction}

\subsection{The Aim of this Work}

The transition leading to a world $\mathrm{Co} 2$ emission level that would not entail a dramatic climate change impact on the actual and future generations is a crucial goal (United Nations, 1992, 1997, 2010, 2015). Its design requires knowledge of the ecological, economic, political and an ethical aspect (Vermeersch, 2005; Johnson, 2009); the first defines the causes and effects of different policies and actions implemented according to the economic and political goals involving a high complexity and a continuous conflict between normative and strategic aspects. To design a transitional trajectory, it is necessary to assign emissions quotas among countries over years and, therefore, to be able to define an international cap and trade system or another equivalent mechanism. The first part of this paper introduces the principles through which design a transition. The second focuses on the transition design method by extending (Bova, 2020). The third shows the data utilized and the fourth indicates the results obtained and the essential features of an international dynamic cap and trade system.

\subsection{The principles and issues to design a transition}

\subsubsection{Discussion}

"The distribution of the right to carbon emission [...] should consider the need for development, population, historical responsibility, the principle of fairness and justice and other factors. As a dominant country of greenhouse gas emission, on the premise of 
sticking to "the principle of common but differentiated responsibilities" (Yang, 2012) We are going to deal with this complexity through three pillars: (1) The developed and developing countries potentially differentiated responsibility, (2) the urgency of the transition and (3) the common responsibility definition and implementation issues.

If there is a limited amount of emissions then the developed countries already absorbed the largest share (see Figure 2 ) and they do have the so-called historical responsibility (The United Nations Development Program, 2008, pp. 40-41); consequently, they are somehow indebted with the other countries (developing and underdeveloped).

Indeed, if developing countries have the right to develop (The project group of International human rights law textbook, 2002, p. 464) a fair allocation of the emission permits should allow them to, such that, since the emissions can be considered indirectly a complementary production factor ${ }^{1}$, there is a trade-off between the growth of developed and developing countries (Mumma \& Hodas, 2008).

The issue is manageable to the extent that the Kuznets curve holds and can be satisfied. It is possible if it allows developing countries to reach the point where a further development entails an emission reduction. If this point is reached then it is possible to keep develop with further benefits in terms of emissions reduction (Uchiyama, 2016; Grossman \& Krueger, 1993; Dasgupta, et al., 2002).

Regarding the historical responsibility, at least three points supports the opposite direction. The first is that the emissions required to develop nowadays are not comparable with the emissions required in the past due to technology changes. Hence, historical responsibility should be somehow pondered. The second point is the observation that a part of the past emission has been absorbed, reducing the "cumulated" responsibility. The third point claims that the possibility to develop for the developing countries has been extremely facilitated by the development of the north of the world. Indeed, by producing new technology even thanks to the emissions generated, it gifted the rest of the world with its innovations such that a part of the historical responsibility can be, by this way, shared for all the humanity. In other words, if the developed world had had no emissions then there would not be such a development and developing margin.

Consequently, the way to deal with this rebus is to start counting from the moment in which the international community recognised the problem and started to implement a shared program. The author individualises in the Kyoto agreement the recognition (1997) and in its implementation (2005) this moment and there the trajectory begin has been fixed. Such a date may be debated, indeed, not all the countries joined or join the Kyoto protocol or the younger Paris agreement, as, for instance, USA and Canada. Can we conclude that the non-participating countries did not or do not recognize their historical responsibility for their $\mathrm{CO} 2$ emissions? More likely, they just considered or consider the terms of these agreements unacceptable (indeed, some of them joined and then quitted). The fact that they decided to deal with such a responsibility trough a different scheme do not neglect the recognition of the responsibility in a first place.

Since to limit the emissions is a responsibility versus the future generations and since it is

${ }^{1}$ Given a production function that for each quantity produced emits a positive amount $f(q)$ then to limit $f(q)$ means to limit q as well. Clearly, the reduction of emissions key is in the technology of the production adopted. According to the Kuznets curve we may indirectly derive that the higher the production the higher the emission until a quantity produce $\mathrm{q}^{*}$ after which the emission per unit start do decline. 
mostly a world problem then we do need both a common threshold and an individual responsibility for each country 'results (Posner \& Sustein, 2008; Green, 2009). Hence, each country must have its own amount of emission permits while sum of those permits respect the common threshold. In turn, each person is responsible as well through its actions and its consumptions. Hence, the emissions should be allowed on a per capita base and governments should be indirectly responsible for their people. Eventually, these permits should be defined for a population in the initial implementation moment and they should not change as the population changes. Indeed, population growth or decline is an intergenerational issue as well and it does depend on the actual population choice. Such a choice, especially for developing countries, is generally viewed as optimal and ethical if the population is maintained constant or decline. Let me explain it in two steps.(1) A naive wellbeing maximization $\sum U(x)$ would entail, with a finite amount of resources $\mathrm{M}$, the maximum population growth since the sum of utility has a first-order derivative positive and a second-order negative: $\sum_{i=1}^{i=n_{1}} U\left(\frac{M}{n_{1}}\right)>\sum_{i=1}^{i=n_{2}} U\left(\frac{M}{n_{2}}\right)$ if $n_{1}>n_{2}$. This is known as the repugnant conclusion (Parfit, 1984). (2) However, according to the Kuznets curve only beyond a given level of utility $U$ the impact (allowed emissions $\mathrm{M}$ ) declines and we do know that: $d U / d n<0$ and $\frac{d M(n) / d n}{n}>0$ such that if the population rises then the utility declines and the impact (M) grows; In turn, if $\mathrm{M}$ is constrained to a constant then the Utility declines again, $\mathrm{M}$ again grows again, and we assist to a spiral of wellbeing decrease. If this is true then to maintain the population is one sure way to ensure constant wellbeing while other equilibria would be much harder to manage and would, in any case, require computation strongly depending on the assumptions on the utility function and the Kuznets curve shapes.

By assigning the permits on a per capita base, developing countries would be allowed to emit more at the beginning of the transition and to gradually reduce them while developed countries would gradually reduce from the beginning (see 2.3). Moreover, by introducing a quasi-decentralised dynamic cap and trade system ( see 4.4) the emission trading across countries may, on one hand, contribute to the developing countries gain from their lower emissions and to develop more sustainably, on the other hand, incentivize the developed countries to reduce their emissions or compensate for the difference. Before discussing these implications, it is necessary to define the equity principles such that we analytically determine the equitable transition.

\subsubsection{Right and duty assignment: equity principles and emission threshold}

We may summarize the issue into two main aspects: the intercountry and the intergenerational equity.

In terms of intercountry equity, the equitable emission distribution is assumed to be that where all the countries reach the same cumulated emission per capita level (or an equivalent compensation, see 4.3)

Such a per capita amount is limited since due to the duty to do not compromise the future generations (intergenerational equity). The intergenerational equity is divided in two moments: the dynamic and static equity (see 2.2 and 2.3): The first refers to the transition necessary to reach a situation that allows to each generation to have an equal amount of 
emissions. The transition is assumed to be fair if it entails the same cost or gain in terms of wellbeing for each generation in a given country, where a generation here is reduced, for obvious technical reasons, to the population living in a given year (see 2.2). The transition is considered satisfied if respecting the emission threshold limits the temperature increase to the goal degrees of Celsius in 2050 (OECD, 2012) (see 4.1). Hence, the period considered will be 2005-2050.

The goal of two degree Celsius and the consequent 450 ppm involves a high complexity and it is debated (Hansen, et al., 2013). Three main aspects concur to the authors' choice: (1) The probability that such a ppm concentration leads to a two-degree Celsius change is close to the $50 \%$ involving high risk, (2) it has been argued such a temperature change may be beneficial although not necessarily equitable (Nordhaus, 2018; Gorth, 2018; Bova, 2020) and (3) it is hard to believe that we may reach a ppm concentration lower than 450 (see Figure 8 ). Hence, the authors apply it being aware that it may be updated through future researches.

\section{Method}

\subsection{Methodology}

The baseline is given by Bova $(2019,2020)$ that provides a methodology to evaluate and define an equitable transition. It was applied to the ecological footprint index (Bova, 2020), and this paper can be considered an extension including both the co2 emissions analysis and new dynamic goal and computational solutions. We apply the first two levels of this method: the static intergenerational equity and the inequality of transition. The first is about the degree of inequality in terms of distance to the equitable situation across countries or generations. The second concern the distance to the so-called fair transitional goal ( see 2.3).

\subsection{Static equity}

The static equity enquires the degree of inequality of a given distribution. In this application, it will be applied to the cumulated per capita emissions distribution across countries and the per capita emissions across generations.

\subsubsection{The equivalent number of equals Index}

To measure the degree of static inequality, for both cases, the index of inequality used is the equivalent number of equals (Q) settled with the parameter $b=2$ (Bova, 2019, 2020).

$Q=\left(\frac{\sum x_{i}^{b}}{\left(\sum x_{i}\right)^{b}}\right)^{\frac{1}{1-b}}$

Where $x_{i}$ is the ith element of the distribution, $\mathrm{Q}$ is the equivalent number of equals, and $\mathrm{b}$ its sensibility parameter or inequality aversion. It measures the equivalent number of equals in terms of cumulated emissions that would generate the same wellbeing $(b<1)$ or inequality $(\mathrm{b}=2)$ of the observed distribution. Indeed, it is equivalent to

$\sum x_{i}^{b}=Q\left(\frac{\sum x_{i}}{Q}\right)^{b}$ 
Where the left side is the sum of the utility $(b<1)$ or inequality (for $b=2$ is the Herfindahl index), $\mathrm{Q}$ is the number of equal parts in which the total amount is divided.

In its rescaled version to assume values between 0 and 1 we will apply the rescaled overlapping index $\left(O_{r s}\right)$

$O_{r s}=\frac{Q-1}{n-1}$

Where $\mathrm{n}$ is the number of elements of the distribution.

\subsection{Dynamic equity}

Dynamic equity is reached if the generations respect their transitional goals. Hence, the inequality of transition is given by their distance to the transitional goals. A transitional goal depends on the equitable situation to reach, the time available. Furthermore, it is equitable if it entails that the cost/gain of transition is equally spread across the participating generations that are those necessary to reach the static equity. A transition is necessary whenever there is no possibility to maintain the actual state across the generations (i.e. it is not sustainable). Therefore, a transition is equitable when it leads to a situation from which we can reach and maintain the static intergenerational equity. It needs a threshold that identifies when we move from a transition to a static situation.

\subsubsection{Static and dynamic equity trade-off}

Since the goal assigned is to reach an amount of per capita pollution equal then the transition would generate a higher per intercountry static equity and but a lower intergenerational static equity. The first is true by definition, the transition will end with an across countries equity and progressive achievement of it reducing the distance among countries. The latter is a consequence of the definition of a cumulated emission goal: countries that were emitting too much must reduce their emissions and vice versa.

\subsubsection{The goal of transition}

The cumulated emissions per capita (Cep) for a given country "i" is given by:

$\operatorname{Cep}_{i, t}=\operatorname{Cep}_{i, t_{o}}+\sum_{t=\mathrm{t}_{0}}^{t=t_{1}} \frac{E_{i, t}}{P_{i, t}}$

Where $\mathrm{E}$ is the emission, $\mathrm{P}$ the population, $\mathrm{i}$ represents the country and $\mathrm{t}$ the year (or generation).

The goal of transition " $g$ " is that portion of the allowed emissions that each country has and that allows an equal level of per capita emission at the end of the transition if the population is unchanged. It is defined according to the following formula

$\mathrm{g}_{\mathrm{i}}=\mathrm{Ce}_{\mathrm{i}, 2005, \mathrm{P}_{\mathrm{i}, 2005}}=\frac{\mathrm{E}_{\text {allowed }}}{\sum P_{i, 2005}} P_{i, 2005}$

Were $\mathrm{Ce}_{\mathrm{i}}$ is the cumulated emissions allowed to a given country in the period 2005-2050. It is given by the total emission allowed $E_{\text {allowed }}$ at world level to reach the $450 \mathrm{ppm}$ goal divided among countries according to their population share $\frac{P_{i, t_{0}}}{\sum P_{i, t_{o}}}$. Ce is the country's right and is limited by both the world's duty to do not compromise the future generation and the other countries' right. It is defined according to the population in the moment of the implementation of the agreement (2005) since, otherwise, demographic factors would interfere and the population growth would be incentivized. Hence, the transition 
respecting such a goal leads to intercountry equity if the population is unchanged.

\subsubsection{The transitional goal}

The transitional goal 'tg' is a constant share " $r$ " of the distance to the goal of transition " $g$ " where " $r$ " is the rate of transition (Bova, 2020). Such a rate ensures that each generation participates in the transition that is mathematically infinite without a threshold distinguishing the static and the dynamic equity (i.e. the transition end). The distance to the goal is the cumulated emission performed (Ce) minus the cumulated emission allowed (g). The fair variation of the cumulated emission $\Delta C e_{i, t}$ can, therefore, be defined as $\Delta C e_{i, t}=r\left(g_{i}-C e_{i, t-1}\right)=\operatorname{tg}$

The trajectory is designed assuming that the country respects the transitional goal and that it achieves the goal of transition in y periods. Moreover, it allows for a gradual reduction of the yearly emission over time (i.e. a decline of the cumulated emission growth).

$\sum_{i=1}^{i=y} r\left(g-C p_{i}\right)=g-t h$

The rate of transition allowing for such equity is labelled fair rate. "Th" stands for threshold and is a positive number between 0 and $g$ allowing this equation to have a rate different from one and laying between 0 and 1 . The threshold identifies the value dividing the dynamic equity (transition) to the static equity.

\subsubsection{Threshold implications and the minimum efficiency rate}

We can normalize the equation as follows

$g \sum_{i=1}^{i=y} r\left(1-C p_{i} / g\right)=\mathrm{g}\left(1-\frac{t h}{g}\right) \rightarrow \sum_{i=1}^{i=y} r\left(1-C p_{i} / g\right)=\left(1-\frac{t h}{g}\right)$

In such a way all the cumulated emissions can be compared as they become a share of the allowed emission. Moreover, it entails that the only parameter distinguishing the countries is the ratio th/g. It is also possible to show that, for the limit of th to zero $r$ converges to a finite value. If this is true, then when th is very close to zero $g$ becomes almost irrelevant and the countries may have the same $r$ : it would depend only on $y$. However, if th is arbitrarily close to zero then the fair rate is arbitrarily defined as well. In particular, the closer the ratio to zero the closer $\mathrm{r}$ is to one. Hence, without a criterion to define the threshold, such an approach determines the rate arbitrarily.

Since we have not a criterion to define a threshold, we are going to introduce an alternative approach: the minimum efficiency rate and the double flow.

The minimum efficiency rate is the minimum efficiency improvement that, if juxtaposed with an equivalent saving rate (in terms of the reduction of emissions) would lead both to the goal assigned and to equal emission based on output.

By imposing that each generation reduces its emission for a share "s", it exists an efficiency improvement " $\mathrm{e}$ " that can counterbalance the emission reduction in terms of output and, therefore, in terms of wellbeing.

$$
\left(1-s_{t}\right) E_{t}\left(1+e_{t+1}\right)=E_{t} \Leftrightarrow\left(1-s_{t}\right)^{i} E_{t}\left(1+e_{t+1}\right)^{i}=E_{t} \Leftrightarrow s_{t}=-\frac{e_{t+1}}{1+e_{t+1}}
$$

The share " $s$ " is determined in such a way as to guarantee the achievement of the goal of the transition. Assuming a constant technology increases over time, the cost in terms of output lost is given by the difference between the observed efficiency improvement " $e_{\text {observed }}$ " and the minimum efficiency rate " $\mathrm{e}$ " for a non-wellbeing loss transition "e". 
The absolute cost of the transition between $t_{o}$ and $t_{\text {end }}$ is given by $\sum_{i=t_{o}}^{i=t_{\text {end }}}(1-s)^{i} E_{t_{0}}\left(1+e-e_{\text {observed }}\right)^{i}$

By comparing developing and developed countries it is possible to observe that they have an inverse relationship between "s" and "e". Indeed, for developing (developed) countries "s" is lower (higher), since the emissions per capita are lower (higher), and "e" is lower (higher) since the technology is weaker (stronger). Hence, a transition so defined could be achieved by both.

\subsubsection{Double flow}

Since some countries have such a low emission in 2004, the year from which the computation of the transition starts, to reach their goal they do need to increase them. However, they do need to be able to reduce emissions closer to 2050. Hence, the easiest way to face this issue is the double flow. The double-flow is a transition composed of two blocks, one where the countries with a negative "s" increase their emissions and another where they reduce them. In the first period, these countries can develop by emitting more, in the second they reduce the yearly emissions to gradually become ready for static equity. In our computations, the two blocks were divided exactly in the middle of the transition. Finally, as described later on (see 4.3), any unbalance can still be an opportunity through the dynamic cap and trade system where each country can follow the best strategy known the cost and the benefit to follow the transition or do even better.

\section{Data}

For our data set we collected 1) Global CO2 emission data from the US Energy Information Administration 2) per capita emission from the Global change data lab 3) the yearly global PPM data from National centres for environmental information (NCDC) and 4) population from world development indicators (World Bank). ${ }^{2} 3$

\section{Results}

In this section follow the results of the static and dynamic equity and the consequent hypothesis of a decentralised dynamic cap and trade system. The result follows the methodology introduced while the the maximum quantity of the overall emissions allowed is summarized below and its estimation is furtherly explained in the appendix. Each country has a quota of such an overall emission allowance according to its population share of the world population in 2005.

Table 1: PPM goal and emissions allowed

\begin{tabular}{|c|c|c|}
\hline $\begin{array}{c}\text { Average ppm in 2004 } \\
\text { Goal ppm in 2050 }\end{array}$ & $\begin{array}{c}\text { Total emissions allowed } \\
\text { (in the regression the emissions are divided by } 10^{9} \text { ) } \\
176.8\end{array}$ & 450
\end{tabular}

2 https://www.eia.gov/international/data/world

${ }^{3}$ https://data.worldbank.org/indicator/SP.POP.TOTL. 


\section{$\triangle P P M$ and estimated $\triangle P P M$}

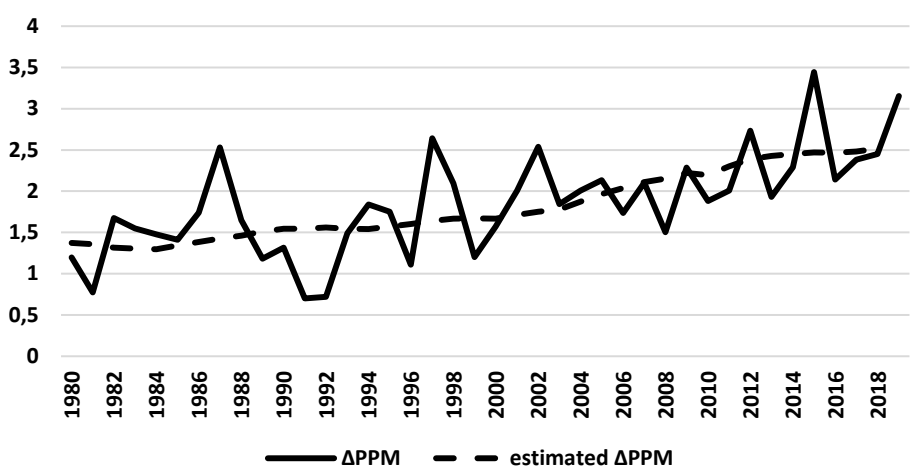

Figure 1: Variation and estimated variation of CO2 part per million (PPM)

\subsection{Static equity}

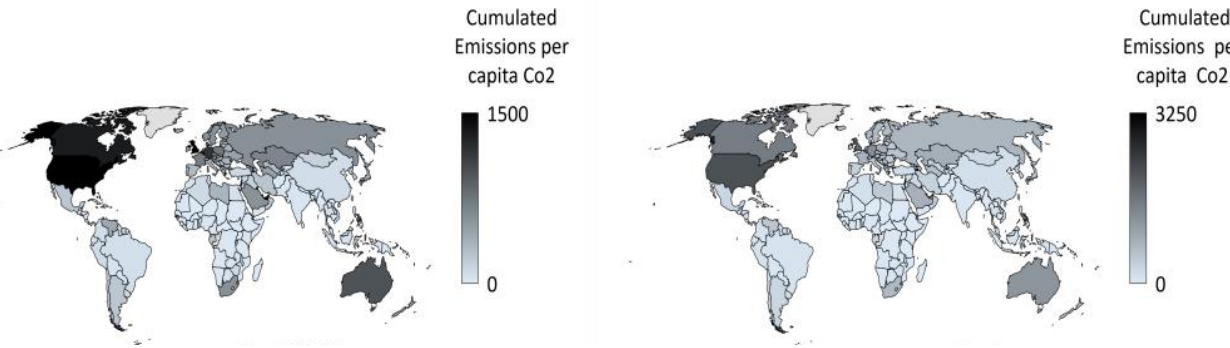

Figure 2: Cumulated emissions per capita (left) in 2005 (right) in 2017

\section{Inter countries equity (Ors, cumulated emissions 1980-2017)}

0,35

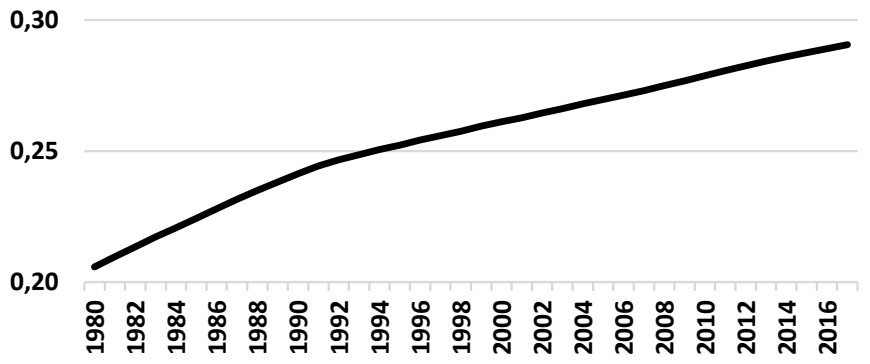

Figure 3: Inequality computed yearly on Cumulated per capita emissions between 1980 and 2017 
Intergenerational static equity (1980-2017)

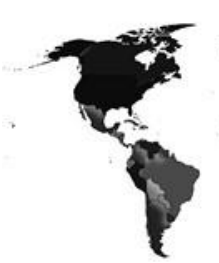

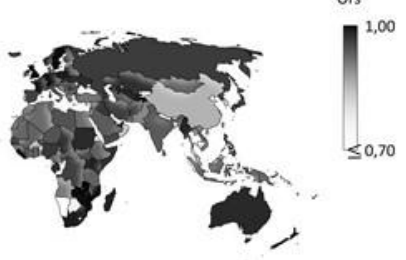

Intergenerational static equity (2005-2017)

ors
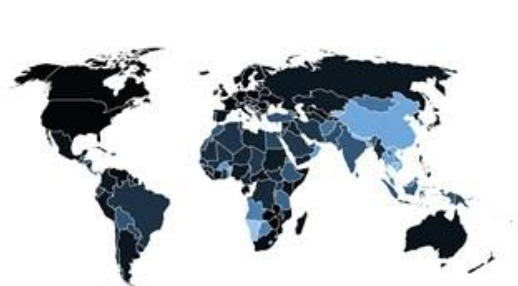

Ors

1,00

0,97

$\leq 0,90$

Figure 4: Intergenerational equity in terms of per capita emissions (left) 1980-2017 (right) 2005-2017

\subsubsection{Comment}

The high concentration of the cumulated co 2 emission showed by the low intercountry equality index Ors in 1980 is mostly due to the cumulated emission of a few developed countries. These countries showed a high intergenerational static equity that is the sign that the emission is not largely changed over time and in particular, it is not changed in 2005-2017. Hence, the improvement of Ors is due to the increased emission of some developing country such as China, India and Brazil. Moreover, developed countries are exhausting or already exhausted (see later) their emission quota such that this intergenerational equity must be interpreted as a resistance to the transition. However, there are non-developed countries that have both low cumulated emissions and high intergenerational equity that may compensate for the potentially unfair behaviour of developed countries and earn by doing so. Such compensation is analysed in 4.3

\subsection{Dynamic equity: Transition}
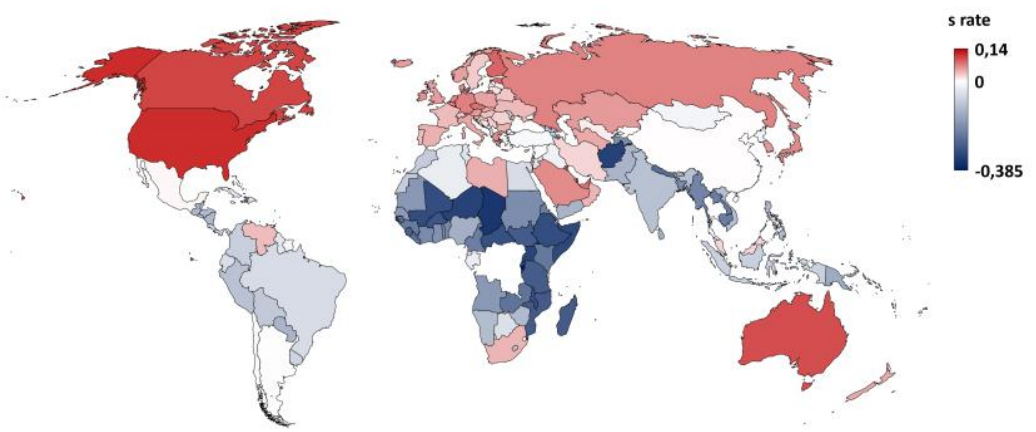

Figure 5: $s$ rates in 2005

In 2005 the countries are divided into three blocks, those who need to reduce their emissions (red), those who may maintain their actual emissions (white) and those which can have a double-flow (an initial increase of the emissions until 2027 and then a decrease). The distinction is, in fact, quite coincident to those between developed, developing and underdeveloped countries. 


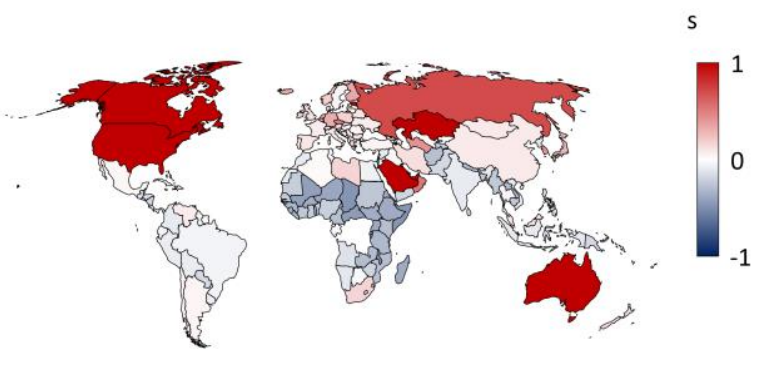

Figure 6: $s$ rates in 2017

This plot shows the same information of the previous one but it is updated to the data of 2017. The differences among countries exploded fast showing, in particular, that North America, Australia, Russia, Kazakhstan and the United Arab Emirates need a tax of saving required equal or close to one. In turn, this entails that they already emitted almost or more than what was allowed and, as such, they are already indebted. Europe, Central and South Africa and the south-west part of Asia are aligned with the transition being neither debtor nor creditor. Africa is composed mostly by creditor countries.

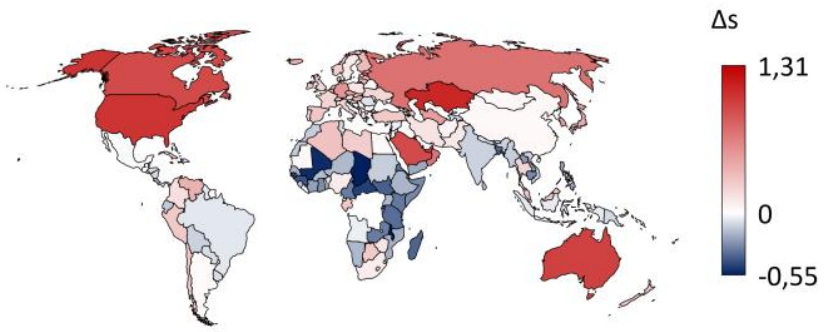

Figure 7: s rate variations between 2005 and 2017

The variation shows which countries accumulated debt (red) and which countries accumulated a credit (blue) or who is consistent with the transition (white). Most of the developed world is indebted, most of the developing countries are aligned with the transition and most of the underdeveloped countries are creditors. Such a situation allows to identify, and it will be discussed better later, which country can benefit or lose with a cap-and-trade system.

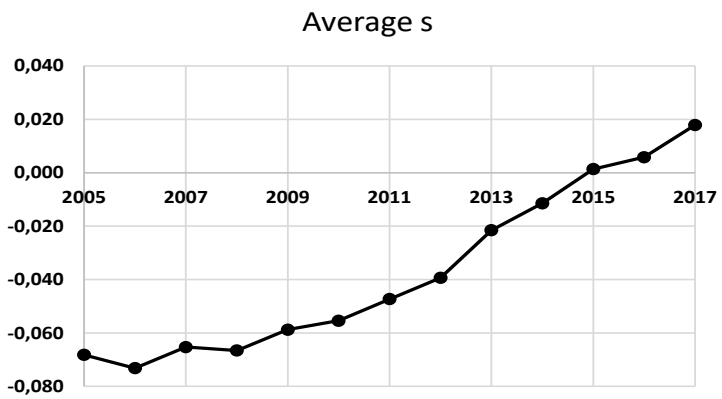

Figure 8: The average s rate of countries over time 
The average "s" rate increase shows that, on average, the countries' performances are becoming worst over time. While at the beginning on average countries could increase their emissions, since 2015 an emissions' reduction is required.

\subsection{A dynamic cap and trade across countries and generations}

\section{Cumulated Credits and Debts in 2018}

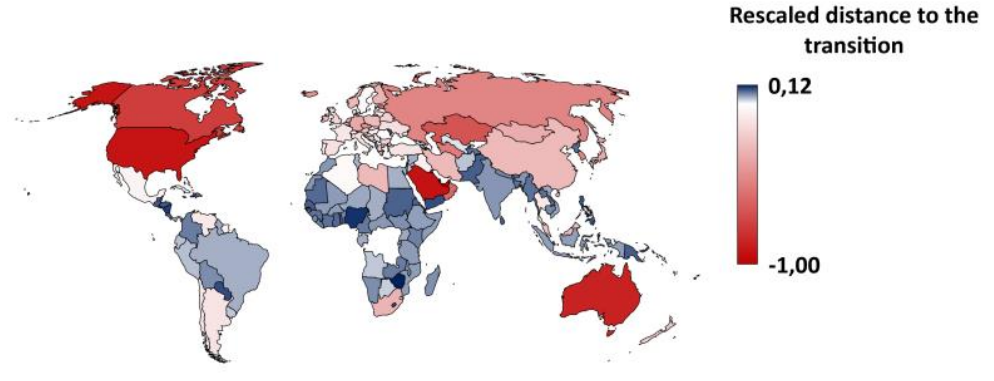

Figure 9: Credits and debts cumulated in 2018. Debt $=$ Red, Credit $=$ Blue. (Countries below -1: Babrain -1,23; Brunei -1,25; Qatar -2,55; Trinidad and Tobago -2,24; the United Arab Emirates -1,19.). The rescaled distance is the sum of the distances to the fair transition divided by the goal.

Without intervention, the emission allowed to respect the 2 degrees goal are expected to be reached largely before 2050. If this will be true, it will imply two inequalities: (1) one imposed on the future generations that would have to furtherly reduce their emissions and that will suffer the climate change consequences and (2) one on the countries that respected their transitional goal (generally the poorest) imposing a burden on their development by furtherly unfairly limiting their emissions. While future generations can hardly be compensated since the previous already dead or retired, a system of compensation is desirable to cope with the second point. For the intercountry equity, a system as the cap and trade properly adjusted may work. It would be composed of the following components

1) A dynamic cap for each country (or region) determined yearly by the allowed cumulated emissions that the transition design determines. This would allow the dynamic intergenerational equity inside a country and, as the mechanism is respected, the static intercountry equity and the future static intergenerational equity. Moreover, the initial allocation of the permits is equity based and this contribute to avoid well known limits of the cap-and-trade system (Maloney \& Yandle, 1984; Hans, 1984).

2) A quasi-decentralised system: decentralised since each country (or region) would have their cap-and-trade system and "quasi" since the different system would be allowed to trade the emissions according to their deviance to their cap to compensate for the difference. This allows for compensation across countries for any deviance from the equitable path leading to the intercountry equity. In turn, to impose a cost on the deviances allows to protect the future generations of both the country and the world.

3) Each decentralised system can self-regulate to maximize its capacity to reach the goal according to the needs of the specific contest where it is applied satisfying the very 
different traditions and heterogeneous mechanism needs (Michaelowa, et al., 2019). Concluding, the achievement of a target is possible only if the agents involved recognizes it and, then, if they decide to pursue it. Approaching the CO2 transition in terms of equity entails that there is a clear target (equity) that may generate participation and commitment. The mean proposed to govern the transition, the quasi-decentralised cap and trade system, is a tool able to achieve cost effectiveness, as cap and trade system does, and to improve the efficiency deriving from determination of the caps by introducing a trade margin. Moreover, the possibility to trade the deviations from the cap entails that some countries with high rate of transition are enormous facilitated to reach their goal and, consequently, their chance to join the system and perform the transition increases remarkably. In turn, this increases the chance of a common success.

\section{Conclusion}

The paper studied the design of the Co2 transition to reach, on the one hand, both an intercountry and an intergenerational equity by discussing the principles adopted by the United Nations and the literature, on the other, a consequent quasi-decentralized cap and trade system that would allow governing such a transition was considered.

It argued that intercountry equity is reached for an equal cumulated emission per capita. To reach this level and to allow to all the generations to have the same per capita emissions amount (static intergenerational equity), we do need a transition that, as such, generates an initial static intergenerational inequality to allow to the future generations to maintain an equitable situation. Such a transition is equitable (dynamic intergenerational equity) if a) the cost and benefits of the transition are equally shared across the generations b) it allows to reach the minimum goal of a temperature increase below the two degrees Celsius. Consequently, we applied and modified the techniques of intergenerational equity and inequality of transition using the world data of $\mathrm{Co} 2$ emissions.

The empirical data shows that intercountry equity is improving over years, however, this is due to the increased emissions of many developing countries (in particular China, India and Brazil) while the developed world is quite stable. Such stability is highlighted since, in most of the developed world, there is a high static intergenerational equity and it can be interpreted as a resistance to the transition.

In terms of transition, we introduced the concept of minimum efficiency rate of transition. This is the rate of efficiency improvement that would allow the country to both reach the intergenerational equity and share the cost/benefit of the transition fairly across the participating generations.

The data shows that in terms of transition, among the developed countries, only Europe is behaving properly while the rest of the countries (U.S.A., Canada, Russia, the United Emirates, Australia) accumulated debt with the rest of the world. Africa, India, South America, and Indonesia are the areas where most of the countries have a credit. Such credit should entail or the possibility to emit more or the possibility to sell their emission permits. Indeed, once the trajectory of the transitions is defined for each country, then it is possible to establish a quasi-decentralised cap and trade system. Such a theoretical mechanism would fix a yearly amount of cumulated emissions allowed to each region (cap) that could be trade their permits reaching both flexibility (caps trade) and cost effectiveness (cap and 
trade).

Finally, the data showed indicates that the challenge to achieve both intercountry and intergenerational equity demands a strong commitment and is becoming harder and harder suggesting that new tools, approaches, and motivations are needed. However, it is argued that a clear equity-based goal and a flexible cost-effective governing mechanism may facilitate the participation to international agreements coping with $\mathrm{Co} 2$ emissions and, by this way, increase our chances to achieve both intercountry and intergenerational equity.

\section{References}

Bova, D. M., 2019. A measure of intergenerational equality: introduction. In: E. Di Bella et al., ed. A.I.Q.U.A.V.. Genova: University of Genova Press, pp. 101-107.

Bova, D. M., 2020. Intergenerational equity and transitional inequality measurement: techniques and empirics. European Journal of Sustainable Development, 9(2), pp. 129-149.

Dasgupta, S., Laplante, B., Wang, H. \& Wheeler, D., 2002. Confronting the environmental Kuznets Curve. The journal of economic perspectives, 16(1), pp. 147-168.

Goodwin, P., Follows, M. J. \& Williams, R. G., 2008. Analytical relationships between atmospheric carbon dioxide, carbon emissions, and ocean processes. Global biochemical cycles, Volume 22, p. BG3030.

Gorth, M. W., 2018. Integration of climate change into lung-run macroeconomic analysis- on the nobel prize of William D. Nordhaus. Springer Berlin beidelberg.

Green, B. A., 2009. Lessons from the Montreal Protocol: Guidance for the next international climate change agreement. Environmental Law, 39(2), p. 281.

Grossman, G. M. \& Krueger, A. B., 1993. Environmental impacts of a north american free trade agreement. In: P. Graber, ed. The Mexico - U.S. freetrade agreement. Cambridge: MIT press, pp. 13-56.

Hansen, J. et al., 2013. Assessing "Dangerous Climate Change": Required Reduction of Carbon Emissions to Protect Young People, Future Generations and Nature. PLOS ONE, 8(12), p. e81648.

Hans, R., 1984. Market power and transferable property rights. Quartely Journal of economics 99, No.4, pp. 75365.

Johnson, S., 2009. Climate change and Global Justice: Crafting fair solutions for nations and peoples. Harvard environmental law review, 33(2), pp. 297-301.

Kheshgi, H. S., Smith, S. J. \& Edmonds, J. A., 2005. Emissions and atmospheric co2 stabilization: Long-term limits and paths. Mitigation and Adaptation Strategies for Global Change, Volume 10, pp. 213-220.

Maloney, M. \& Yandle, B., 1984. Estimation of the cost of air pollution control regulation. Journal of environmental economics and management, No.3, pp. 244-63.

Michaelowa, A., Shishlow, I. \& Brescia, D., 2019. Evolution of international carbon markets: lessons for the Paris Agreement. WIREs Climate Change, 10(e613), pp. 1-24.

Mumma, A. \& Hodas, D., 2008. Designing a Global post-Kyoto climate change protocol that advances human development. Georgetown international environmental law review, 20(4), p. 619.

Nordhaus, W. D., 2018. Projections and uncertainties about climate change in an era of minimal climate policies. American economic journal: economic policy, 10(3), pp. 333-360.

OECD, 2012. OECD environmental outlook to 2050, s.l.: OECD.

Parfit, D., 1984. Reasons and persons. Oxford: Oxford University Press.

Posner, E. A. \& Sustein, C. R., 2008. Climate change justice. Georgetown law journal, 96(4), pp. 1565-1612.

Raupach, M. R. et al., 2011. The relationship between peak warming and cumulative CO2 emissions, and its use to quantify vulnerabilities in the carbon-climate human system. Tellus B: Chemical and Physical Meteorology, 63(2), pp. 145-164.

The project group of International human rights law textbook, 2002. International buman rights law textbook. Beijing: China political and law press.

The United Nations Development Program, 2008. Human Development report 2007/2008, s.1.: The United Nations.

Uchiyama, K., 2016. Emprirical analysis of the environmental Kuznets curve. In: S. i. Economics, ed. Environmental Kuznets Curve Hypothesis and Carbon Dioxide Emissions. Tokyo: Springer, pp. 31-45.

United Nations, 1992. The united nations framework convention on climate change. s.l., UNFCCC. 
United Nations, 1997. The Kyoto Protocol to the Framework Convention on Climate change. Kyoto, UNFCCC.

United Nations, 2010. United nations climate change conference. s.l., UNFCCC.

United Nations, 2015. Transforming our world: the 2030 agenda for sustainable development, s.l.: United Nations A/RES/70/1.

van Vuuren, D. P. \& Riahi, K., 2011. The relationship between short-term emissions and long-term concentration targets: a letter. Climatic Change, Volume 104, pp. 793-801.

Vermeersch, E., 2005. Reading the Kyoto protocol: ethical aspects of the convention on climate change. Euborun Uitgeverij B.V.: Delf.

Yang, Z. L., 2012. The right to carbon emission: a new right to development. American journal of climate change, 1(1), pp. 108-116.

\section{Appendix: Estimation of the world cumulated emission limit}

To define the transition, we need to determine the level of cumulated emissions allowed respecting the goal of two degrees of temperature increase. Hence, we seek for the Co2 ppm and world emission relationship. We multiplied the world population for the world per capita emission ${ }^{4}$ to obtain the yearly emission and then we run regression between the ppm of Co2 variation $\left(\triangle P P M_{t}\right.$, dependent variable) and the emission of the previous year $\left(E_{t-1}\right.$, independent variable).

$\triangle P P M_{t}=\beta E_{t-1}+u$

We tested different models and the best are described by the following tables. They included two lags, the year before ( $\mathrm{t}-1)$ and two years before $(\mathrm{t}-2)$ and both with and without robust errors. The results of such an analysis must be considered prudently since the data set available had a little sample of 39 observations. Moreover, many different aspects concur to the real result, on one hand, the temperature is affected by many other elements, other greenhouse gasses, the albedo effects, the change in clouds and rains etc, on the other, the absorption and accumulation of the $\mathrm{Co} 2$ in the atmosphere is affected by many factors, from the ocean absorption to the deforestation, that is unlikely to be all stable over time and with constant absorption coefficients (Goodwin, et al., 2008) (Raupach, et al., 2011). Finally, the relationship is not only short-term (van Vuuren \& Riahi, 2011) (Kheshgi, et al., 2005). Hence, we present the results necessary for us to proceed while we are aware that they do require constant update and further research.

For the single explanatory variable (yearly emissions), the coefficient $\beta$ is significant with $5 \%$ level for each model and the R2 is, not surprisingly, high. The adjusted R 2 rewards the robust error approach and make it clear that the lag $\mathrm{t}-2$ is the better. From the Akaike criterion, we controlled for the loss of information that is good for each model and supports more the t-2 lag. Also, the Durbin-Watson value confirms for each model a positive correlation. By analysing better, the lag $\mathrm{t}-2$ we can see the normality of the errors. The results are not surprising since the $\mathrm{Co} 2$ concentration in the air is a function of the quantity of emitted Co2. The fact that each lag has the same coefficient chooses this to determine the total emissions allowed easier although the little sample.

\footnotetext{
${ }^{4}$ Our sample includes between the 93 and the 99 percent of the world emissions and the 98 percent of the world population.
} 
Table 2: Models

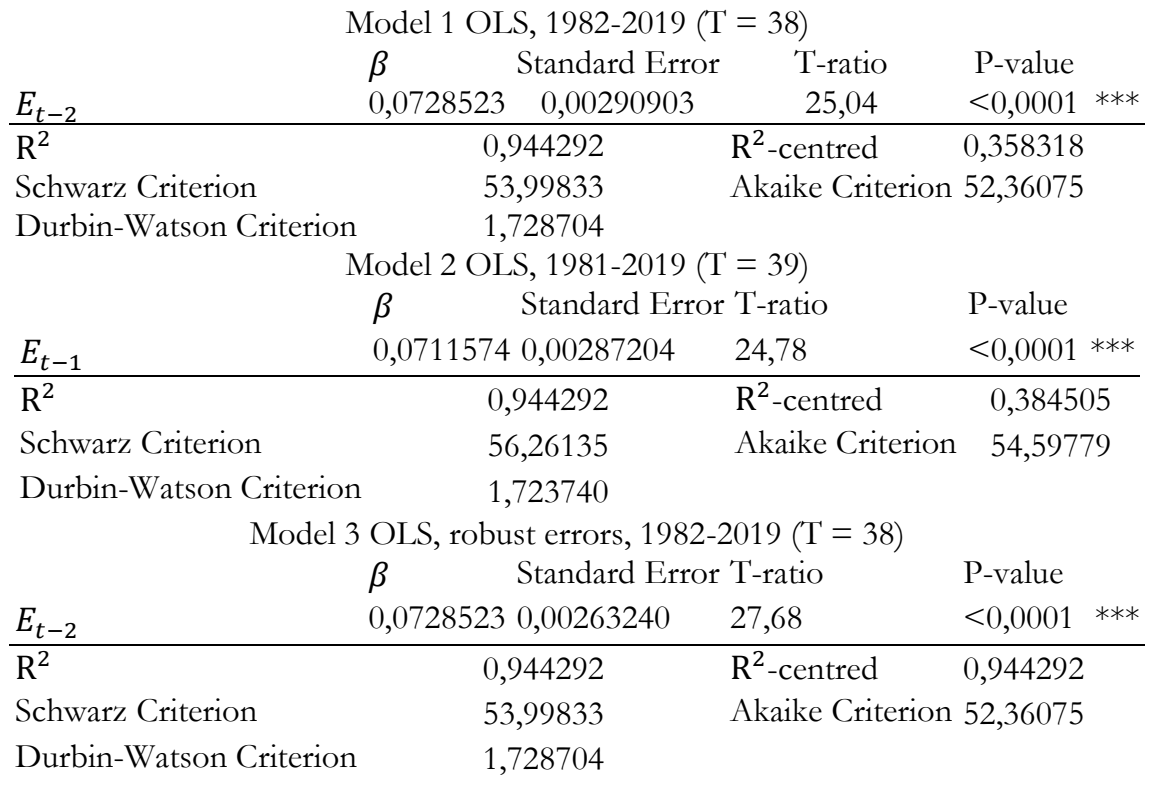

Model 4 OLS, robust errors, 1981-2019 $(\mathrm{T}=39)$

\begin{tabular}{|c|c|c|c|}
\hline & Standard Er & T-ratio & $\mathrm{P}$-value \\
\hline$E_{t-1}$ & $0,07115740,00259861$ & 27,38 & $<0,0001 \quad * * *$ \\
\hline $\mathrm{R}^{2}$ & 0,941704 & $\mathrm{R}^{2}$-centred & 0,941704 \\
\hline Schwarz Criterion & 56,26135 & Akaike Criterion & 54,59779 \\
\hline Durbin-Watson Criterion & 1,723740 & & \\
\hline
\end{tabular}

Table 3: PPM goal and emissions allowed

Average ppm in

2004

376.8
Goal ppm in 2050 450
Total emissions allowed (in the regression the emissions are divided by $10^{9}$ ) $1058.9 \cdot 10^{9}$

\section{$\triangle P P M$ and estimated $\triangle P P M$}

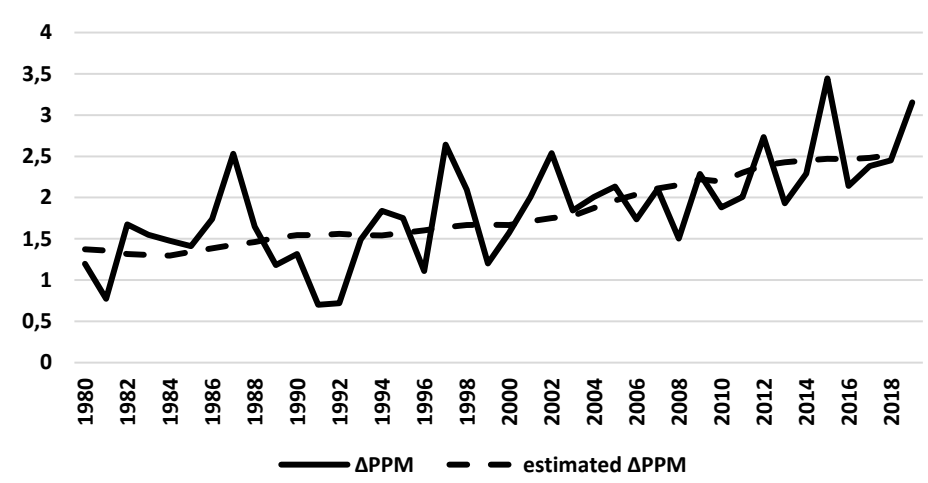

Figure 10: Observed and estimated variation of Co2 ppm. Model 3 


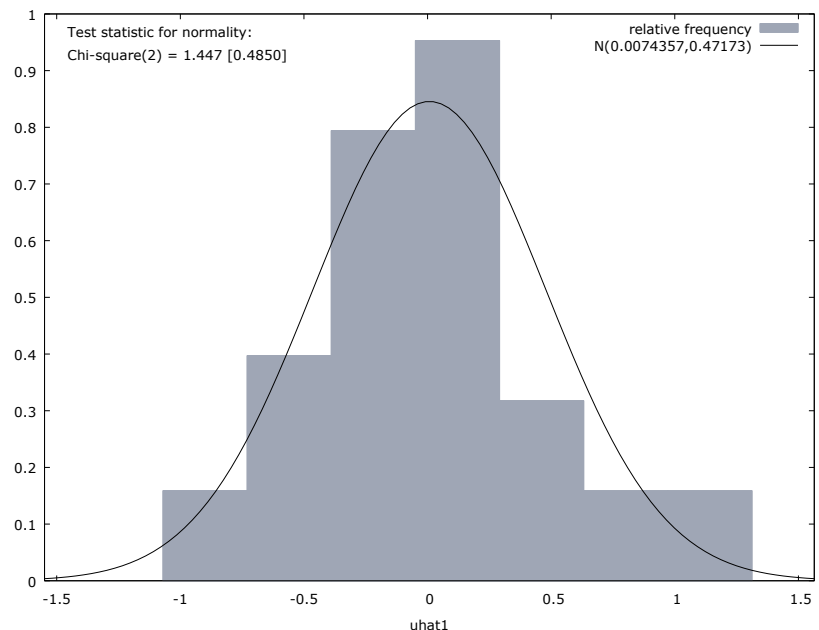

Figure 11: Residual normality. Uhat1=Residuals. Model 3 